\title{
Surface polaritons in symmetry planes of biaxial crystals
}

\author{
A N Furs, V M Galynsky and L M Barkovsky \\ Department of Theoretical Physics, Belarussian State University, Fr. Skarina Ave. 4, \\ Minsk 220050, Belarus \\ E-mail: Barkovsky@bsu.by
}

Received 3 February 2005, in final form 3 August 2005

Published 31 August 2005

Online at stacks.iop.org/JPhysA/38/8083

\begin{abstract}
The problem of the surface polariton existence in symmetry planes of nonmagnetic biaxial crystals is studied theoretically. The plane interface of such a crystal and a semi-infinite isotropic medium is considered. With the use of the integral formalism formulated in our earlier work, the dispersion equation is derived for the polaritons under consideration. The existence conditions for the dispersion equation solutions are obtained in the form of algebraic inequalities for principal values of inverse dielectric permittivity tensors. If these conditions are satisfied, then excitation of surface waves is possible along the allowed propagation directions, which constitute sectors in the interface plane. Exact expressions are obtained that determine location of these sectors with respect to the symmetry axes of the crystal.
\end{abstract}

PACS numbers: $42.25 . \mathrm{Bs}, 71.36 .+\mathrm{c}$

\section{Introduction}

Recently, research has focused closely on polariton states in condensed matter including surface polaritons. Such states may be characterized by a term 'half light, half matter' [1], inasmuch as for polariton excitations the properties of light and matter are intertwined in a remarkable manner.

Surface polaritons, being the electromagnetic excitations localized near the interface at a distance of the order of wavelength, can be tentatively divided into two classes. The first of them represents well-studied surface waves at the interface of isotropic media with opposite signs of dielectric permittivities and strong frequency dispersion [2]. Surface plasmon polaritons are among them. Not long ago, a second class of surface polaritons was theoretically predicted $[3,4]$, which appear owing to anisotropy of contacting materials and are called singular surface electromagnetic waves. Unlike surface electromagnetic waves of the first class, singular surface polaritons can be excited in bianisotropic materials with positive definite 
dielectric permittivity and magnetic permeability tensors $\varepsilon$ and $\mu$ when dispersion is little. In particular, such surface polaritons appear in anisotropic dielectric and magnetic media, as well as gyrotropic and Faraday media $[5,6]$. The allowed propagation directions of polaritons form sectors in the interface plane. The greater the anisotropy of one or two contacting media, the greater the angular width of these sectors. Optical anisotropy manifests itself to a considerable extent for singular surface polaritons when the interface is formed by different cuts of the same uniaxial crystal [7-9]. Such an interface can be formed if the uniaxial crystal is divided in half by a plane passing through its optical axis and then parts of the crystal are turned around each other. As a result, their optical axes are made crosswise in the interface plane.

Surface electromagnetic waves at the interface of a uniaxial crystal and an isotropic medium were studied in $[3,4,10]$. The more complicated case of surface waves in the symmetry planes of biaxial crystals was considered in [11]. There, the dispersion relation was found for such waves in the form of a system of algebraic equations. But in view of the complexity of the obtained equations, the authors restricted themselves to solving the system only for the case of weakly anisotropic crystals using expansions in terms of small anisotropy parameters. Approximate formulae were found which determine position of the sectors of the allowed propagation directions for polaritons. Meanwhile, singular surface polaritons typically reveal themselves just in strongly anisotropic crystals.

The aim of the present work is a more thorough study of surface polaritons in the symmetry planes of biaxial crystals with arbitrary degree of anisotropy without the use of any approximations. In the process, we apply the general integral approach worked out originally in the theory of surface elastic waves (see [12] and references therein) and then extended by us to surface polaritons [13-15]. The essence of this approach is in a representation of Maxwell's equations by a system of matrix equations with six-dimensional vectors involved in it. These vectors are composed of tangential components of electric and magnetic strength vectors at the interface. As a result, the dispersion equation for surface waves is expressed in terms of the surface impedance tensors, which in turn are calculated as a product of the special tensors in the integral representation (see section 2).

Advantages of the integral approach are the following. The dispersion equations are derived uniformly regardless of complexity of the constitutive equations for contacting linear media [15]. Moreover, for arbitrary cuts of anisotropic and bianisotropic media, the dispersion equations can in principle be derived analytically with the use of modern systems of computer algebra. They are presented in a general form $F(v)=0$, where $v=\omega /(c k)$ is a reduced frequency of a surface wave. As a rule, $F(v)$ is monotonic function of $v$. It allows comparatively simple analysis of existence of the dispersion equation solutions.

While existence conditions for surface electromagnetic waves are being established, it is important to find the so-called limiting frequencies of these waves [15]. Calculation of these for biaxial crystals is based on study of the refraction surface sections (sections 3 and 4). Further, in sections 5 and 6, the surface impedance tensors are found and necessary existence conditions are established for surface polaritons in the symmetry planes of biaxial crystals. Derivation of the dispersion equation and analysis of existence of its solutions for arbitrary relations involving the material parameters of the contacting biaxial crystal and isotropic medium are given in section 7. Proceeding to the limit of surface waves in uniaxial crystals is discussed in section 8 .

Here, we study surface polaritons only in non-magnetic, non-chiral transparent biaxial crystals with real symmetric dielectric permittivity tensors. Consideration of chiral or absorbing biaxial crystals is possible in principle with the use of general approach developed in [15] but it is more complicated. This is a topic of further publications. Note that simple 
introduction of chirality for isotropic media (Faraday media) when the dielectric tensor is Hermitian leads to fundamentally new physical effects with respect to surface polaritons. In particular, one of them is unidirectional propagation of surface polaritons - if the surface wave can propagate in some direction $\boldsymbol{b}$ along the interface, then it cannot propagate in the opposite direction $-\boldsymbol{b}$ (see $[5,6]$ for details).

In this paper, we use the following notation of operations with three-dimensional scalars, vectors and tensors. The scalar (internal) product of vectors $\boldsymbol{u}$ and $\boldsymbol{v}$ is marked as $\boldsymbol{u} \boldsymbol{v}$, the vector (external) product as $\boldsymbol{u} \times \boldsymbol{v}$ and the tensor product as $\boldsymbol{u} \otimes \boldsymbol{v}$. The scalar product of a tensor $\beta$ and a vector $\boldsymbol{u}$ is a vector $\boldsymbol{w}=\beta \boldsymbol{u}$ with components $w_{i}=\beta_{i j} u_{j}$ (summation is implied over repeating indices). A dual antisymmetric pseudotensor of the second rank $\boldsymbol{u}^{\times}$ can be associated with vector $\boldsymbol{u}$ in accordance with formula $\left(\boldsymbol{u}^{\times}\right)_{i k}=e_{i j k} u_{j}$, where $e_{i j k}$ is the Levi-Civita pseudotensor and $u_{j}$ are Cartesian components of vector $\boldsymbol{u}$. For such tensors, the relations $\boldsymbol{u}^{\times} \boldsymbol{v}=\boldsymbol{u} \times \boldsymbol{v}, \boldsymbol{v} \boldsymbol{u}^{\times}=\boldsymbol{v} \times \boldsymbol{u}$ take place, i.e. vector product of vectors $\boldsymbol{u}$ and $\boldsymbol{v}$ is replaced by scalar product of dual tensor $\boldsymbol{u}^{\times}$and vector $\boldsymbol{v}$.

\section{General case of surface electromagnetic wave propagation at the interface of anisotropic dielectric media}

Consider first a general case of surface electromagnetic waves at the interface of anisotropic dielectric media.

Let $\boldsymbol{q}$ be a unit normal to the interface of anisotropic media directed from the medium with permittivity tensor $\varepsilon=\varepsilon(\omega)$ to the medium with tensor $\varepsilon^{\prime}=\varepsilon^{\prime}(\omega)$, where $\varepsilon$ and $\varepsilon^{\prime}$ are real symmetric tensors and $\omega$ is the frequency of the surface wave. The Cartesian axis $z$ is directed along $\boldsymbol{q}$, coordinate plane $z=0$ aligning with the interface. Field distribution in both media can be presented by a superposition of the two inhomogeneous partial waves. For instance, in the medium $z<0$ characterized by tensor $\varepsilon$, we have

$$
\begin{aligned}
& \boldsymbol{H}(\boldsymbol{r}, t)=\sum_{s=1}^{2} C_{s} \boldsymbol{H}_{s}^{0} \exp \left[\mathrm{i} k\left(\boldsymbol{b}+\eta_{s} \boldsymbol{q}\right) \boldsymbol{r}-\mathrm{i} \omega t\right]=\sum_{s=1}^{2} C_{s} \boldsymbol{H}_{s}^{0} \exp \left[\mathrm{i} \omega\left(\frac{1}{c} \boldsymbol{m}_{s} \boldsymbol{r}-t\right)\right], \\
& \boldsymbol{E}(\boldsymbol{r}, t)=\sum_{s=1}^{2} C_{s} \boldsymbol{E}_{s}^{0} \exp \left[\mathrm{i} k\left(\boldsymbol{b}+\eta_{s} \boldsymbol{q}\right) \boldsymbol{r}-\mathrm{i} \omega t\right]=\sum_{s=1}^{2} C_{s} \boldsymbol{E}_{s}^{0} \exp \left[\mathrm{i} \omega\left(\frac{1}{c} \boldsymbol{m}_{s} \boldsymbol{r}-t\right)\right],
\end{aligned}
$$

where a unit vector $\boldsymbol{b}$ determines the propagation direction of the wave along the interface plane, $k$ is the projection of the wave vector to $\boldsymbol{b}, \boldsymbol{H}_{s}^{0}$ and $\boldsymbol{E}_{s}^{0}$ are vector amplitudes of the partial waves and $C_{s}$ are weight factors $(s=1,2)$. Complex coefficients $\eta_{s}\left(\operatorname{Im} \eta_{s}<0\right)$ characterize decay of the surface wave when moving away from the interface. They are included in the complex refraction vectors

$$
\boldsymbol{m}_{s}=\frac{1}{v}\left(\boldsymbol{b}+\eta_{s} \boldsymbol{q}\right), \quad s=1,2,
$$

where reduced frequency $v=\omega /(c k)$ represents the phase velocity of the surface wave in units of $c$ (velocity of light in vacuum). Fields $\boldsymbol{H}^{\prime}(\boldsymbol{r}, t), \boldsymbol{E}^{\prime}(\boldsymbol{r}, t)$ in the second medium $(z>0)$ are also described by equations of type (1) with the change of symbols $\boldsymbol{H}_{s}^{0}, \boldsymbol{E}_{s}^{0}, C_{s}, \eta_{s}, \boldsymbol{m}_{s}$ to those with a prime. But here the decay coefficients $\eta_{s}^{\prime}$ are subject to the condition $\operatorname{Im} \eta_{s}^{\prime}>0$.

Boundary conditions for the waves (1) take the form

$$
\boldsymbol{H}_{\tau}^{0}=\boldsymbol{H}_{\tau}^{\prime 0}, \quad \boldsymbol{q} \times \boldsymbol{E}^{0}=\boldsymbol{q} \times \boldsymbol{E}^{\prime 0},
$$

where

$$
\boldsymbol{H}_{\tau}^{0}=\sum_{s=1}^{2} C_{s} \boldsymbol{H}_{s \tau}^{0}, \quad \boldsymbol{q} \times \boldsymbol{E}^{0}=\sum_{s=1}^{2} C_{s} \boldsymbol{q} \times \boldsymbol{E}_{s}^{0}
$$




$$
\boldsymbol{H}_{\tau}^{\prime 0}=\sum_{s=1}^{2} C_{s}^{\prime} \boldsymbol{H}_{s \tau}^{\prime 0}, \quad \boldsymbol{q} \times{\boldsymbol{E}^{\prime}}^{0}=\sum_{s=1}^{2} C_{s}^{\prime} \boldsymbol{q} \times{\boldsymbol{E}^{\prime 0}}_{s},
$$

and subscript $\tau$ denotes tangential components of the vector fields (projection of the field vectors to the interface plane).

Resulting values of the electric and magnetic strengths at the interface $\boldsymbol{q} \times \boldsymbol{E}^{0}$ and $\boldsymbol{H}_{\tau}^{0}$ $\left(\boldsymbol{q} \times \boldsymbol{E}^{\prime 0}\right.$ and $\left.\boldsymbol{H}_{\tau}^{\prime 0}\right)$ connect between themselves linearly through a planar surface impedance tensor $\gamma\left(\gamma^{\prime}\right)[16]$ :

$$
\boldsymbol{q} \times \boldsymbol{E}^{0}=\gamma \boldsymbol{H}_{\tau}^{0}, \quad \boldsymbol{q} \times \boldsymbol{E}^{\prime 0}=\gamma^{\prime} \boldsymbol{H}_{\tau}^{\prime 0} .
$$

Eliminating vector variables from (2) and (3), we obtain a dispersion equation for the surface polaritons

$$
\left(\overline{\gamma-\gamma^{\prime}}\right)_{t}=0
$$

where $\left(\overline{\gamma-\gamma^{\prime}}\right)_{t}$ is a trace of the tensor adjoined to $\gamma-\gamma^{\prime}{ }^{1}$

In [13-15], it was shown that anti-Hermitian surface impedance tensors can be calculated by formulae ${ }^{2}$

$$
\gamma=\frac{1}{v} Q^{-}(-\mathrm{i} I-S), \quad \gamma^{\prime}=\frac{1}{v} Q^{\prime-}\left(\mathrm{i} I-S^{\prime}\right) .
$$

Tensors $Q$ and $S$ have an integral representation

$$
Q=-\frac{1}{\pi} \int_{0}^{\pi}\left(e_{2} e_{2}\right)^{-} \mathrm{d} \phi, \quad S=-\frac{1}{\pi} \int_{0}^{\pi}\left(e_{2} e_{2}\right)^{-}\left(e_{2} e_{1}\right) \mathrm{d} \phi .
$$

Integrals in equations (6) include tensorial bilinear forms of arbitrary vector arguments $u$ and $\boldsymbol{v}$ defined by

$$
(\boldsymbol{u v})_{k n}=c_{k l m n} u_{l} v_{m},
$$

where the fourth rank tensor $c_{k l m n}$ is introduced

$$
c_{k l m n}=-\frac{\left(\overline{\varepsilon^{-1}}\right)_{l m} a_{k} a_{n}+v^{2} I_{k r} e_{r l i} \varepsilon_{i j}^{-1} e_{j m p} I_{p n}}{\boldsymbol{a} \varepsilon^{-1} \boldsymbol{a}-v^{2}}+v^{2} b_{l} b_{m} I_{k n}
$$

and $\boldsymbol{a}=\boldsymbol{b} \times \boldsymbol{q}$. Vectors $\boldsymbol{e}_{1}$ and $\boldsymbol{e}_{2}$ in (6) are expressed in terms of the vectors $\boldsymbol{b}$ and $\boldsymbol{q}$

$$
e_{1}=\boldsymbol{b} \cos \phi+\boldsymbol{q} \sin \phi, \quad \boldsymbol{e}_{2}=-\boldsymbol{b} \sin \phi+\boldsymbol{q} \cos \phi
$$

Tensors $Q^{\prime}$ and $S^{\prime}$ can also be calculated by formulae (6) and (7) with the replacement of $\varepsilon$ with $\varepsilon^{\prime}$ in (7).

The left-hand side of dispersion equation (4) is a function of the reduced frequency $v$. Its solution $v=v_{\mathrm{S}}$ describes a surface electromagnetic wave localized close to the interface if $0<v_{\mathrm{S}}<\hat{v}_{\mathrm{L}}$, where $\hat{v}_{\mathrm{L}}=\min \left(v_{\mathrm{L}}, v_{\mathrm{L}}^{\prime}\right)$ and $v_{\mathrm{L}}, v_{\mathrm{L}}^{\prime}$ are the so-called limiting frequencies of surface electromagnetic waves in each contacting medium. These frequencies have a great importance for determining existence conditions of surface polaritons and can be found geometrically using the refraction surface sections by a plane passing through vectors $\boldsymbol{b}$ and $\boldsymbol{q}$ (see $[14,15]$ and next section). Furthermore, tensors $Q$ and $S(6)$ exist only if $v \in\left[0, v_{\mathrm{L}}\right)$, and $Q^{\prime}$ and $S^{\prime}$ exist only if $v \in\left[0, v_{\mathrm{L}}^{\prime}\right)$. For an isotropic medium with permittivity $\varepsilon^{\prime}$, the limiting frequency is $\nu_{\mathrm{L}}^{\prime}=1 / \sqrt{\varepsilon^{\prime}}$. If anisotropic dielectric with permittivity tensor $\varepsilon$ contacts with

1 By definition, the adjoined tensor is $\bar{\beta}_{i j}=\frac{1}{2} e_{k l j} e_{m n i} \beta_{k m} \beta_{l n}$ [17]. The trace $\bar{\beta}_{t}$ of the tensor adjoined to planar tensor $\beta(\beta \boldsymbol{q}=\boldsymbol{q} \beta=0)$ is equal to the determinant of the matrix representing $\beta$ in a subspace orthogonal to $\boldsymbol{q}$.

2 Symbol $^{-}$denotes pseudoinversion operation of the planar tensors, i.e. inversion in a subspace orthogonal to $q$. By definition, $Q Q^{-}=Q^{-} Q=I$, where $I=-\boldsymbol{q}^{\times^{2}}=1-\boldsymbol{q} \otimes \boldsymbol{q}$ is a projective operator to the boundary plane [18, 19]. 
an isotropic medium, then the necessary existence condition can be expressed as follows (see [14]):

$$
-\mathrm{i} \lim _{\nu \rightarrow v_{\mathrm{L}}} \boldsymbol{a} \gamma \boldsymbol{a}>0
$$

Under condition (9), it is possible to select a value of permittivity $\varepsilon^{\prime}$ of the isotropic medium in order to allow propagation of a surface electromagnetic wave along a given direction $\boldsymbol{b}$.

\section{Refraction surface sections of biaxial crystals}

We apply the results of the previous section to the surface electromagnetic waves at the interface of biaxial crystal with permittivity tensor

$$
\varepsilon=a_{1}^{-1} c_{1} \otimes c_{1}+a_{2}^{-1} c_{2} \otimes c_{2}+a_{3}^{-1} c_{3} \otimes c_{3}
$$

and isotropic medium with permittivity $\varepsilon^{\prime}$. In formula (10), unit vectors $c_{1}, c_{2}, c_{3}$ are directed along the principal axes of the tensor $\varepsilon$, and $a_{1}^{-1}=\varepsilon_{1}, a_{2}^{-1}=\varepsilon_{2}, a_{3}^{-1}=\varepsilon_{3}$ are the principal values of $\varepsilon$ at the wave frequency $\omega$. To denote inverse permittivity of the isotropic medium, we use the symbol $a^{\prime}=1 / \varepsilon^{\prime}$. All material parameters $a_{1}, a_{2}, a_{3}, a^{\prime}$ are supposed to be real and positive.

Let a plane crystal cut be perpendicular to one of the principal axes of the tensor $\varepsilon$, i.e. it coincides with one of the crystallographic symmetry planes of the crystal. Hereinafter when using the term 'biaxial crystal' with respect to surface electromagnetic waves, we presume that the cut plane is oriented in such a manner. Without loss of generality, we can get an axis directed along $c_{2}$ as a normal to the cut plane and assume that vectors $c_{1}, c_{2}, c_{3}$ produce a right-hand triple and, in addition, $a_{1}>a_{3}$.

Surface electromagnetic wave propagation direction is specified by an angle $\alpha$ measured from the vector $c_{1}: b=c_{1} \cos \alpha+c_{3} \sin \alpha$. Vectors $\boldsymbol{b}$ and $\boldsymbol{a}=\boldsymbol{b} \times \boldsymbol{q}$ lie in the cut plane, so

$$
\boldsymbol{c}_{1}=\boldsymbol{b} \cos \alpha-\boldsymbol{a} \sin \alpha, \quad \boldsymbol{c}_{2}=\boldsymbol{q}, \quad \boldsymbol{c}_{3}=\boldsymbol{b} \sin \alpha+\boldsymbol{a} \cos \alpha .
$$

To determine the dependence of limiting frequencies $v_{\mathrm{L}}$ for surface electromagnetic waves in a biaxial crystal on angle $\alpha$, we need to study the refraction surface of this crystal and its section by a reference plane (plane passing through vectors $\boldsymbol{b}$ and $\boldsymbol{q}$ ). The refraction vector of the body electromagnetic wave with phase normal $n\left(n^{2}=1\right)$ is determined by the equation $\boldsymbol{m}=n \boldsymbol{n}$, where $n$ is a wave refractive index. Refraction surface constitutes a locus of extreme points of the refraction vectors, whose beginnings are aligned in the reference point $O$. Vectors $\boldsymbol{m}$ satisfy an equation $\operatorname{det}\left(\boldsymbol{m}^{\times} \varepsilon^{-1} \boldsymbol{m}^{\times}+1\right)=0$ or in the expanded form (see [17])

$$
\boldsymbol{m}^{2} \boldsymbol{m} \varepsilon \boldsymbol{m}+\boldsymbol{m}\left(\bar{\varepsilon}-\bar{\varepsilon}_{t}\right) \boldsymbol{m}+\operatorname{det} \varepsilon=0 .
$$

Taking into account vector $\boldsymbol{m}$ decomposition $\boldsymbol{m}=m_{1} \boldsymbol{c}_{1}+m_{2} \boldsymbol{c}_{2}+m_{3} \boldsymbol{c}_{3}$ and relations

$$
\begin{aligned}
& \bar{\varepsilon}=a_{2}^{-1} a_{3}^{-1} c_{1} \otimes c_{1}+a_{1}^{-1} a_{3}^{-1} c_{2} \otimes c_{2}+a_{1}^{-1} a_{2}^{-1} c_{3} \otimes c_{3}, \\
& \bar{\varepsilon}_{t}=a_{2}^{-1} a_{3}^{-1}+a_{1}^{-1} a_{3}^{-1}+a_{1}^{-1} a_{2}^{-1}, \quad \operatorname{det} \varepsilon=a_{1}^{-1} a_{2}^{-1} a_{3}^{-1},
\end{aligned}
$$

equation (12) takes the form

$$
\begin{aligned}
\left(m_{1}^{2}+m_{2}^{2}+m_{3}^{2}\right) & \left(a_{2} a_{3} m_{1}^{2}+a_{1} a_{3} m_{2}^{2}+a_{1} a_{2} m_{3}^{2}\right) \\
& -\left(a_{2}+a_{3}\right) m_{1}^{2}-\left(a_{1}+a_{3}\right) m_{2}^{2}-\left(a_{1}+a_{2}\right) m_{3}^{2}+1=0 .
\end{aligned}
$$

Equation (13) determines the relation between refraction vector components $m_{1}, m_{2}, m_{3}$ and is an equation for the refraction surface of body electromagnetic waves in biaxial crystals. This surface is closed and consists of two sheets, inasmuch as two waves can propagate along a given direction $\boldsymbol{n}$ with two different phase velocities. Vector components $m_{1}, m_{2}, m_{3}$ enter 


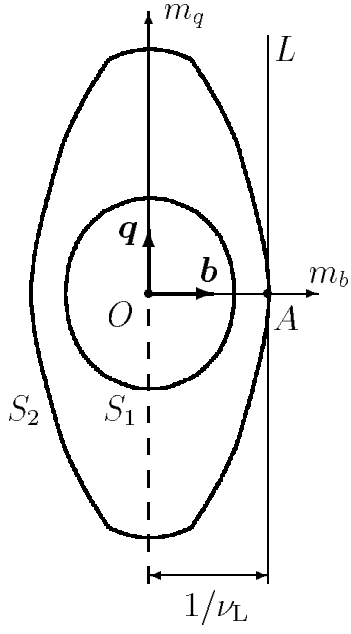

(a)

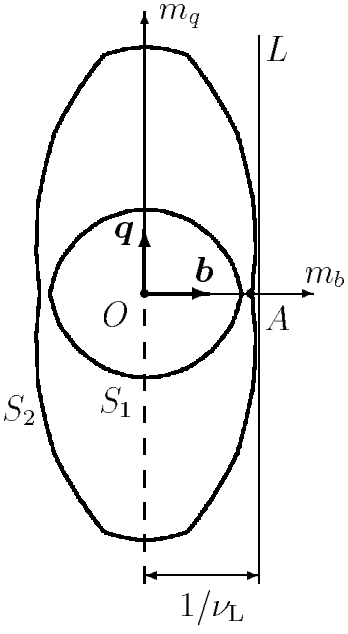

$(b)$

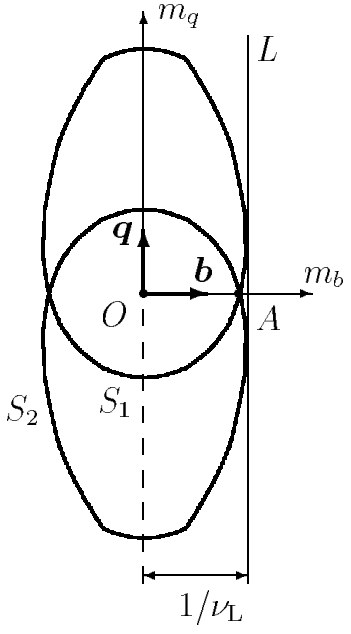

(c)

Figure 1. Refraction surface sections of the biaxial crystals with the reference planes: (a) $a_{1}=0.7, a_{2}=0.9, a_{3}=0.1, \alpha=50^{\circ} ;$ (b) $a_{1}=0.9, a_{2}=0.7, a_{3}=0.1, \alpha=50^{\circ}$; (c) $a_{1}=0.9, a_{2}=0.7, a_{3}=0.1, \alpha=\alpha_{0}=60^{\circ}$.

into (13) in even powers so the refraction surface is centrosymmetrical, and coordinate planes passing through basis vectors $c_{1}$ and $c_{2}, c_{2}$ and $c_{3}, c_{3}$ and $c_{1}$ are symmetry planes of the refraction surface.

The reference plane with unit normal $\boldsymbol{a}$ forms an angle $\alpha$ with vector $c_{1}$. In view of the surface refraction symmetry, it is enough to study its reference plane section only at angle range $0 \leqslant \alpha \leqslant \pi / 2$.

Now we find equations of the section curves. The left- and right-hand sides of equations (11) scalarly multiplied by the vector $\boldsymbol{m}$ take the form

$m_{1}=m_{b} \cos \alpha-m_{a} \sin \alpha, \quad m_{2}=m_{q}, \quad m_{3}=m_{b} \sin \alpha+m_{a} \cos \alpha$,

where $m_{b}, m_{q}, m_{a}$ are components of $\boldsymbol{m}$ in $(\boldsymbol{b}, \boldsymbol{q}, \boldsymbol{a})$ basis. For any vector in the reference plane, its component along $\boldsymbol{a}$ equals zero: $m_{a}=0$. If we substitute (14) into (13) and suppose $m_{a}=0$, we obtain the following curve equation:

$$
\left(m_{b}^{2}+m_{q}^{2}\right)\left(a_{2} d m_{b}^{2}+a_{1} a_{3} m_{q}^{2}\right)-\left(a_{2}+d\right) m_{b}^{2}-\left(a_{1}+a_{3}\right) m_{q}^{2}+1=0
$$

where

$$
d=a_{1} \sin ^{2} \alpha+a_{3} \cos ^{2} \alpha .
$$

Note that $a_{3} \leqslant d \leqslant a_{1}$. The section consists of inner $S_{1}$ and outer $S_{2}$ curves. If the reference plane passes through an optical axis (direction along which two eigenwaves propagate with the same phase velocity), then they have common points. Since equation (15) is a fourth-order equation with respect to $m_{b}$ and $m_{q}$, then any straight line in the section plane may cross the curves at not more than four points. This implies that inner curve must be convex, while outer curve may be either convex or concave (see figure 1).

Let refraction vector $\boldsymbol{m}$ in section plane be directed at angle $\theta$ to vector $\boldsymbol{b}$. Then, its components equal

$$
m_{b}=|\boldsymbol{m}| \cos \theta, \quad m_{q}=|\boldsymbol{m}| \sin \theta,
$$


where $|\boldsymbol{m}|=\sqrt{\boldsymbol{m}^{2}}=\sqrt{m_{b}^{2}+m_{q}^{2}}$ is a modulus of vector $\boldsymbol{m}$. Now we substitute (17) into (15) and write equation (15) in the polar coordinates $|\boldsymbol{m}|$ and $\theta$ :

$\boldsymbol{m}^{4}\left(a_{2} d \cos ^{2} \theta+a_{1} a_{3} \sin ^{2} \theta\right)-\boldsymbol{m}^{2}\left[\left(a_{2}+d\right) \cos ^{2} \theta+\left(a_{1}+a_{3}\right) \sin ^{2} \theta\right]+1=0$.

From (18), we find equations of the curves in the reference plane:

$$
\begin{aligned}
m^{2}=n^{2}= & \frac{1}{2}\left(a_{2} d \cos ^{2} \theta+a_{1} a_{3} \sin ^{2} \theta\right)^{-1}\left\{\left(a_{2}+d\right) \cos ^{2} \theta+\left(a_{1}+a_{3}\right) \sin ^{2} \theta\right. \\
& \left. \pm\left[\left[\left(a_{2}+d\right) \cos ^{2} \theta+\left(a_{1}+a_{3}\right) \sin ^{2} \theta\right]^{2}-4\left(a_{2} d \cos ^{2} \theta+a_{1} a_{3} \sin ^{2} \theta\right)\right]^{\frac{1}{2}}\right\},
\end{aligned}
$$

where plus sign corresponds to the outer curve $S_{2}$ and minus sign corresponds to the inner curve $S_{1}$. Then, we return in equations (19) to the variables $m_{b}$ and $m_{q}$ considering (17) and introducing notation $\kappa=m_{q}^{2} / m_{b}^{2}=\tan ^{2} \theta$ :

$$
\begin{aligned}
& 2 m_{b}^{2}(1+\kappa)\left(a_{2} d+a_{1} a_{3} \kappa\right)\left\{a_{2}+d+\left(a_{1}+a_{3}\right) \kappa\right. \\
& \left.\quad \pm\left[\left[a_{2}+d+\left(a_{1}+a_{3}\right) \kappa\right]^{2}-4(1+\kappa)\left(a_{2} d+a_{1} a_{3} \kappa\right)\right]^{\frac{1}{2}}\right\}^{-1}=1 .
\end{aligned}
$$

It is obvious (see (20)) that curves $S_{1}$ and $S_{2}$ are symmetrical relative to the coordinate axes directed along vectors $\boldsymbol{b}$ and $\boldsymbol{q}$.

Limiting frequencies $v_{\mathrm{L}}$ of the surface waves can be found geometrically by the following way. Let $L$ be a straight line parallel to vector $\boldsymbol{q}$ and originally located at infinity, which moves towards $-\boldsymbol{b}$ until the first contact with the section curve $S_{2}$ of the outer sheet of the refraction surface (figure 1). Then, the limiting frequency $\nu_{\mathrm{L}}$ is reciprocal of the distance from the point $O$ to the line $L$, passing through one or several points of tangency. When $v=v_{\mathrm{L}}$, at least one of the coefficients $\eta_{1}, \eta_{2}$ in (1) becomes real and now corresponds to the body partial wave. In order to establish location of the contact by the straight line $L$, it is important to investigate parts of the section curve $S_{2}$ close to the coordinate axis $m_{b}$, i.e. when $\kappa$ is small. We choose in equation (20) the upper sign corresponding to curve $S_{2}$ and retain only terms with first order of $\kappa$, so at $a_{2}>d$, we have

$$
d m_{b}^{2}+\frac{a_{1} a_{3}-d\left(a_{1}+a_{3}-a_{2}\right)}{a_{2}-d} m_{q}^{2}=1
$$

and at $a_{2}<d$,

$$
a_{2} m_{b}^{2}+\frac{a_{1} a_{3}-a_{2}\left(a_{1}+a_{3}-d\right)}{d-a_{2}} m_{q}^{2}=1 .
$$

Thus, parts of the section curve close to the $b$-direction approximately seem as a second-order curve with a general equation $h_{b} m_{b}^{2}+h_{q} m_{q}^{2}=1$. This curve crosses $m_{b}$-axis at point $A$ at the distance $O A=1 / \sqrt{h_{b}}$. Depending on the sign of the coefficient $h_{q}$ of $m_{q}^{2}$, equations (21) and (22) correspond either to an ellipse $\left(h_{q}>0\right)$ or to a hyperbola $\left(h_{q}<0\right)$. In the first case, the section curve $S_{2}$ is a convex curve at small values of $m_{q}$ and in the second case, the curve is concave.

Consider the following cases of relations involving parameters $a_{1}, a_{2}$ and $a_{3}$ contained in tensor $\varepsilon$ (10):

$$
\text { (i) } a_{2} \geqslant a_{1}>a_{3}, \quad \text { (ii) } a_{1}>a_{2}>a_{3}, \quad \text { (iii) } a_{1}>a_{3} \geqslant a_{2} \text {. }
$$

If relation (i) is satisfied, then $a_{2}>d$ at $\alpha \neq \frac{\pi}{2}$ and we analyse equation (21). It is easy to show that the coefficient of $m_{q}^{2}$ will be positive:

$$
a_{1} a_{3}-d\left(a_{1}+a_{3}-a_{2}\right)>a_{1} a_{3}-a_{1}\left(a_{1}+a_{3}-a_{2}\right)=a_{1}\left(a_{2}-a_{1}\right) \geqslant 0 ;
$$

therefore, outer section curve is convex at small $m_{q}$. Distance $O A$ equals $1 / \sqrt{d}$ and depends on $\alpha$ (i.e. depends on the position of the reference plane). Similarly, if relation (iii) is satisfied, then $a_{2}<d$ at $\alpha \neq 0$, so equation (22) causes the curve $S_{2}$ to be convex at small $m_{q}$ too and 
to cross the coordinate axis at distance $O A=1 / \sqrt{a_{2}}$ which does not depend on $\alpha$. Finally, for the relation (ii), we have $a_{2} \gtrless d$. Let angle $\alpha_{0}$ be determined by equation $a_{2}=d$ (see also (16))

$$
\sin \alpha_{0}=\sqrt{\frac{a_{2}-a_{3}}{a_{1}-a_{3}}}
$$

and angles $\alpha_{1}$ and $\alpha_{2}$ be determined by the equations $a_{1} a_{3}-d\left(a_{1}+a_{3}-a_{2}\right)=0$ and $a_{1} a_{3}-a_{2}\left(a_{1}+a_{3}-d\right)=0$, respectively,

$$
\sin \alpha_{1}=\sqrt{\frac{a_{3}\left(a_{2}-a_{3}\right)}{\left(a_{1}+a_{3}-a_{2}\right)\left(a_{1}-a_{3}\right)}}, \quad \sin \alpha_{2}=\sqrt{\frac{a_{1}\left(a_{2}-a_{3}\right)}{a_{2}\left(a_{1}-a_{3}\right)}},
$$

resulting in vanishing of coefficient $h_{q}$ of $m_{q}^{2}$ in equations (21) and (22). Then, in the case (ii), an inequality $\alpha_{1}<\alpha_{0}<\alpha_{2}$ is satisfied, and at $0 \leqslant \alpha<\alpha_{1}$ or $\alpha_{2}<\alpha \leqslant \frac{\pi}{2}$, a part of the curve $S_{2}$ close to the point $A$ is convex, but at $\alpha_{1}<\alpha<\alpha_{2}$ it is concave. If $\alpha<\alpha_{0}$, then $O A=1 / \sqrt{d}$, otherwise $O A=1 / \sqrt{a_{2}}$.

Thus, when relation (ii) is satisfied and while angle $\alpha$ is increased from 0 to $\frac{\pi}{2}$, at $\alpha=\alpha_{1}$, convexity of the curve part $S_{2}$ near point $A$ changes to concavity, but at $\alpha=\alpha_{2}$ it is vice versa. Intermediate angle $\alpha_{0}(23)$ corresponds to the contact of the inner and outer section curves at point $A$ (see figure $1(c)$ ). In that case, the section reference plane passes through the optical axis of the biaxial crystal whose direction is given by the unit vector $c^{\prime}=c_{1} \cos \alpha_{0}+c_{3} \sin \alpha_{0}$. Owing to symmetry of the refraction surface, a vector $c^{\prime \prime}$ (vector of other optical axis) is obtained by replacement of $\alpha_{0}$ with $-\alpha_{0}$ in the last equation: $\boldsymbol{c}^{\prime \prime}=\boldsymbol{c}_{1} \cos \alpha_{0}-\boldsymbol{c}_{3} \sin \alpha_{0}$. Note that vectors $c^{\prime}$ and $c^{\prime \prime}$ are included in the Fedorov axial representation of the permittivity tensor for biaxial crystal [17]

$$
\varepsilon^{-1}=a_{2}+\frac{a_{1}-a_{3}}{2}\left(c^{\prime} \otimes c^{\prime \prime}+c^{\prime \prime} \otimes c^{\prime}\right)
$$

\section{Limiting frequencies of surface electromagnetic waves in biaxial crystals}

It is easy to calculate limiting frequency $v_{\mathrm{L}}$ of a surface electromagnetic wave in the case when the outer section curve $S_{2}$ is a convex curve near the point $A$ (see figure $1(a)$ ). Then, the straight line $L$ contacts curve $S_{2}$ at the point $A$ and limiting frequency $v_{\mathrm{L}}$ equals reciprocal of length of the line segment $O A$. Particularly, as is set forth in previous section, $v_{\mathrm{L}}=\sqrt{d}$ at any $\alpha$ when relations (i) are satisfied, or in case (ii) with conditions $0 \leqslant \alpha \leqslant \alpha_{1}$ satisfied. Next, $v_{\mathrm{L}}=\sqrt{a_{2}}$ at any $\alpha$ when relations (iii) are satisfied, or in case (ii) with satisfied conditions $\alpha_{2} \leqslant \alpha \leqslant \frac{\pi}{2}$.

If curve $S_{2}$ is concave close to the point $A$ and line $L$ contacts this curve at two points (conditions (ii) with $\alpha_{1}<\alpha<\alpha_{2}$ ), then limiting frequencies need to be calculated individually. We differentiate equation (15) with respect to $m_{q}$ and take into account that for a contact point $\mathrm{d} m_{b} / \mathrm{d} m_{q}=0$. As a result, we have

$$
m_{q}^{2}=\frac{a_{1}+a_{3}}{2 a_{1} a_{3}}-\frac{a_{2} d+a_{1} a_{3}}{2 a_{1} a_{3}} m_{b}^{2},
$$

with the assumption that $m_{q} \neq 0$. After substitution of (25) in equation (15) for the section curves and taking into consideration that the limiting frequency $v_{\mathrm{L}}$ is calculated by means of coordinate $m_{b}$ as $\nu_{\mathrm{L}}=1 / m_{b}$, we get

$\left(a_{1}-a_{3}\right)^{2} v_{\mathrm{L}}^{4}-2\left[\left(a_{1}+a_{3}\right)\left(a_{2} d+a_{1} a_{3}\right)-2 a_{1} a_{3}\left(a_{2}+d\right)\right] v_{\mathrm{L}}^{2}+\left(a_{2} d-a_{1} a_{3}\right)^{2}=0$. 
Table 1. Limiting frequencies $v_{\mathrm{L}}$ of surface electromagnetic waves in biaxial crystals.

\begin{tabular}{llll}
\hline Case & $\begin{array}{l}\text { Relations } \\
\text { between } a_{1}, a_{2}, a_{3}\end{array}$ & Angle $\alpha$ & Limiting frequency $v_{\mathrm{L}}$ \\
\hline (i) & $a_{2} \geqslant a_{1}>a_{3}$ & $0 \leqslant \alpha<\frac{\pi}{2}$ & $\nu_{\mathrm{L}}=\sqrt{d}$ \\
(ii(a)) & $a_{1}>a_{2}>a_{3}$ & $0 \leqslant \alpha<\alpha_{1}$ & $\nu_{\mathrm{L}}=\sqrt{d}$ \\
(ii(b)) & $a_{1}>a_{2}>a_{3}$ & $\alpha_{1} \leqslant \alpha \leqslant \alpha_{2}$ & $\nu_{\mathrm{L}}=\widetilde{v}_{\mathrm{L}}(27)$ \\
(ii(c)) & $a_{1}>a_{2}>a_{3}$ & $\alpha_{2}<\alpha \leqslant \frac{\pi}{2}$ & $\nu_{\mathrm{L}}=\sqrt{a_{2}}$ \\
(iii) & $a_{1}>a_{3} \geqslant a_{2}$ & $0<\alpha \leqslant \frac{\pi}{2}$ & $\nu_{\mathrm{L}}=\sqrt{a_{2}}$ \\
\hline
\end{tabular}

We denote solutions of biquadratic equation (26) as $\widetilde{\nu}_{\mathrm{L}}$ and finally obtain an expression for the limiting frequency

$$
\begin{aligned}
\widetilde{v}_{\mathrm{L}}^{2}=\frac{1}{\left(a_{1}-a_{3}\right)^{2}} & {\left[\left(a_{1}+a_{3}\right)\left(a_{2} d+a_{1} a_{3}\right)-2 a_{1} a_{3}\left(a_{2}+d\right)\right.} \\
+ & \left.2 \sqrt{a_{1} a_{3}\left(a_{1}-a_{2}\right)\left(a_{2}-a_{3}\right)\left(a_{1}-d\right)\left(d-a_{3}\right)}\right] .
\end{aligned}
$$

Note that selection of the positive sign of the radical in (27) is determined by the requirement of continuity of limiting frequency $\nu_{\mathrm{L}}$ at points $\alpha=\alpha_{1}$ and $\alpha=\alpha_{2}$ (24). Indeed, when $\alpha=\alpha_{1}$ (i.e. $a_{2}=a_{1}+a_{3}-a_{1} a_{3} / d$ ), limiting frequency has to be equal to $v_{\mathrm{L}}=\sqrt{d}$, but when $\alpha=\alpha_{2}$ ( $\left.d=a_{1}+a_{3}-a_{1} a_{3} / a_{2}\right)$, it has to be equal to $\nu_{\mathrm{L}}=\sqrt{a_{2}}$. It is true only if in solution (27) of biquadratic equation (26), the positive sign of the radical is selected.

The obtained values of limiting frequencies $\nu_{\mathrm{L}}$ are charted in table 1, used later for establishing existence conditions of surface waves at the interface of a biaxial crystal and an isotropic medium. From the obtained equations for $v_{\mathrm{L}}$, it is evident that function $v_{\mathrm{L}}=v_{\mathrm{L}}(\alpha)$ is non-decreasing on the interval $\left[0, \frac{\pi}{2}\right]$ and has a limit

$$
v_{\mathrm{L}} \leqslant \min \left(\sqrt{a_{2}}, \sqrt{d}\right) .
$$

Strict inequality in (28) is achieved only in the case (ii) at $\alpha_{1}<\alpha<\alpha_{2}$ and equality is achieved in other cases.

\section{Derivation of the surface impedance tensors $\gamma$ and $\gamma^{\prime}$}

The dispersion equation for surface electromagnetic waves at the interface of a biaxial crystal and an isotropic medium will be obtained by substitution of the surface impedance tensors $\gamma$ and $\gamma^{\prime}$ of the contacting media to formula (4). To find these tensors, we first need to calculate bilinear tensorial forms $\left(e_{2} e_{2}\right)$ and $\left(e_{2} e_{1}\right)$ related to a biaxial crystal, then tensors $\left(e_{2} e_{2}\right)^{-}$and $\left(e_{2} e_{2}\right)^{-}\left(e_{2} e_{1}\right)$, and finally $Q$ and $S(6)$. Tensors $Q^{\prime}$ and $S^{\prime}$ in formula (5) for $\gamma^{\prime}$ are calculated analogously as $Q$ and $S$ with replacement $a_{1}, a_{2}, a_{3} \rightarrow a^{\prime}$ (going from the biaxial crystal to the isotropic medium).

According to (7), general expressions for the bilinear tensorial forms $\left(e_{2} e_{2}\right)$ and $\left(e_{2} e_{1}\right)$ take the coordinate-free form

$\left(\boldsymbol{e}_{2} \boldsymbol{e}_{j}\right)=-\left(\boldsymbol{a} \varepsilon^{-1} \boldsymbol{a}-v^{2}\right)^{-1}\left[\boldsymbol{e}_{2} \overline{\varepsilon^{-1}} \boldsymbol{e}_{j}(\boldsymbol{a} \otimes \boldsymbol{a})+v^{2} I \boldsymbol{e}_{2}^{\times} \varepsilon^{-1} \boldsymbol{e}_{j}^{\times} I\right]+\boldsymbol{e}_{2} \boldsymbol{b} \otimes \boldsymbol{b} \boldsymbol{e}_{j} v^{2} I$,

where $j=1,2$ and $\boldsymbol{e}_{2} \boldsymbol{b} \otimes \boldsymbol{b} \boldsymbol{e}_{j}$ is the dyad $\boldsymbol{b} \otimes \boldsymbol{b}$ multiplied by vector $\boldsymbol{e}_{2}$ from the left and by vector $\boldsymbol{e}_{j}$ from the right. Tensors $\varepsilon^{-1}$ and $\overline{\varepsilon^{-1}}$ in (29) are given by formulae

$$
\varepsilon^{-1}=\sum_{k=1}^{3} a_{k} c_{k} \otimes c_{k}, \quad \overline{\varepsilon^{-1}}=a_{1} a_{2} a_{3} \sum_{k=1}^{3} a_{k}^{-1} c_{k} \otimes c_{k} .
$$


Therefore,

$$
\begin{aligned}
& \boldsymbol{e}_{2} \overline{\varepsilon^{-1}} \boldsymbol{e}_{j}=a_{1} a_{2} a_{3} \sum_{k=1}^{3} a_{k}^{-1} \boldsymbol{e}_{2} \boldsymbol{c}_{k} \otimes \boldsymbol{c}_{k} \boldsymbol{e}_{j}, \\
& I \boldsymbol{e}_{2}^{\times} \varepsilon^{-1} \boldsymbol{e}_{j}^{\times} \boldsymbol{I}=-\sum_{k=1}^{3} a_{k} I\left(\boldsymbol{c}_{k} \times \boldsymbol{e}_{2}\right) \otimes\left(\boldsymbol{c}_{k} \times \boldsymbol{e}_{j}\right) I .
\end{aligned}
$$

Since $I=\boldsymbol{b} \otimes \boldsymbol{b}+\boldsymbol{a} \otimes \boldsymbol{a}$, then

$$
\left(c_{k} \times e_{j}\right) I=I\left(c_{k} \times e_{j}\right)=\left[\left(c_{k} \times e_{j}\right) b\right] b+\left[\left(c_{k} \times e_{j}\right) a\right] a .
$$

By substituting vectors $c_{1}, c_{2}, c_{3}(11)$ and $e_{1}, e_{2}$ (8) into equations (30) and (31), and then by substituting these equations in (29), we obtain decomposition of the forms $\left(e_{2} e_{2}\right)$ and $\left(e_{2} e_{1}\right)$ in the tensor basis $\boldsymbol{b} \otimes \boldsymbol{b}, \boldsymbol{b} \otimes \boldsymbol{a}, \boldsymbol{a} \otimes \boldsymbol{b}, \boldsymbol{a} \otimes \boldsymbol{a}$ :

$$
\begin{aligned}
\left(\boldsymbol{e}_{2} \boldsymbol{e}_{2}\right)=(d- & \left.v^{2}\right)^{-1}\left\{v^{2}\left[\left(d-v^{2}\right) \sin ^{2} \phi+d \cos ^{2} \phi\right] \boldsymbol{b} \otimes \boldsymbol{b}\right. \\
+ & v^{2}\left(a_{1}-a_{3}\right) \sin \alpha \cos \alpha \cos ^{2} \phi(\boldsymbol{b} \otimes \boldsymbol{a}+\boldsymbol{a} \otimes \boldsymbol{b}) \\
& \left.-\left[\left(a_{2}-v^{2}\right)\left(d-v^{2}\right) \sin ^{2} \phi+\left(a_{1} a_{3}-v^{2}\left(a_{1}+a_{3}-d\right)\right) \cos ^{2} \phi\right] \boldsymbol{a} \otimes \boldsymbol{a}\right\} \\
\left(\boldsymbol{e}_{2} \boldsymbol{e}_{1}\right)=\sin \phi & \cos \phi\left(d-v^{2}\right)^{-1}\left\{v^{4} \boldsymbol{b} \otimes \boldsymbol{b}+v^{2}\left(a_{1}-a_{3}\right) \sin \alpha \cos \alpha(\boldsymbol{b} \otimes \boldsymbol{a}+\boldsymbol{a} \otimes \boldsymbol{b})\right. \\
- & {\left.\left[a_{1} a_{3}-\left(a_{2}-v^{2}\right)\left(d-v^{2}\right)-v^{2}\left(a_{1}+a_{3}-d\right)\right] \boldsymbol{a} \otimes \boldsymbol{a}\right\} . }
\end{aligned}
$$

In the subspace orthogonal to vector $\boldsymbol{q}$ (in the interface plane), bilinear tensorial forms $\left(e_{2} e_{2}\right)$ and $\left(e_{2} e_{1}\right)$ can be represented by $2 \times 2$ matrices with elements equal to the coefficients of the decomposition of these forms in the tensor basis. Therefore, the determinant of $\left(e_{2} e_{2}\right)$ which equals the trace of the adjoined tensor $\overline{\left(e_{2} e_{2}\right)}$ is

$$
\begin{aligned}
{\overline{\left(e_{2} e_{2}\right)_{t}}}_{t} & =\left[b\left(e_{2} e_{2}\right) b\right]\left[a\left(e_{2} e_{2}\right) a\right]-\left[b\left(e_{2} e_{2}\right) a\right]\left[a\left(e_{2} e_{2}\right) b\right] \\
& =-v^{2}\left(d-v^{2}\right)^{-1}\left(A \cos ^{4} \phi+B \sin ^{2} \phi \cos ^{2} \phi+C \sin ^{4} \phi\right),
\end{aligned}
$$

where terms in brackets are matrix elements of the form $\left(e_{2} e_{2}\right)$ and

$$
A=a_{1} a_{3}, \quad B=a_{1} a_{3}+a_{2} d-v^{2}\left(a_{1}+a_{3}\right), \quad C=\left(a_{2}-v^{2}\right)\left(d-v^{2}\right) .
$$

The tensor pseudoinverse to $\left(\boldsymbol{e}_{2} \boldsymbol{e}_{2}\right)$ equals

$$
\begin{aligned}
\left(\boldsymbol{e}_{2} \boldsymbol{e}_{2}\right)^{-}=( & \left.A \cos ^{4} \phi+B \sin ^{2} \phi \cos ^{2} \phi+C \sin ^{4} \phi\right)^{-1}\left\{\frac { 1 } { v ^ { 2 } } \left[\left(a_{2}-v^{2}\right)\left(d-v^{2}\right) \sin ^{2} \phi\right.\right. \\
+ & \left.\left(a_{1} a_{3}-v^{2}\left(a_{1}+a_{3}-d\right)\right) \cos ^{2} \phi\right] \boldsymbol{b} \otimes \boldsymbol{b}+\left(a_{1}-a_{3}\right) \sin \alpha \cos \alpha \cos ^{2} \phi \\
& \left.\times(\boldsymbol{b} \otimes \boldsymbol{a}+\boldsymbol{a} \otimes \boldsymbol{b})-\left[\left(d-v^{2}\right) \sin ^{2} \phi+d \cos ^{2} \phi\right] \boldsymbol{a} \otimes \boldsymbol{a}\right\} .
\end{aligned}
$$

Multiplying $\left(\boldsymbol{e}_{2} \boldsymbol{e}_{2}\right)^{-}(35)$ by $\left(\boldsymbol{e}_{2} \boldsymbol{e}_{1}\right)(33)$, we obtain

$$
\begin{aligned}
\left(e_{2} e_{2}\right)^{-}\left(e_{2} e_{1}\right) & =\sin \phi \cos \phi\left(A \cos ^{4} \phi+B \sin ^{2} \phi \cos ^{2} \phi+C \sin ^{4} \phi\right)^{-1} \\
& \times\left\{v^{2}\left[\left(a_{2}-v^{2}\right) \sin ^{2} \phi+\left(a_{1}+a_{3}-d\right) \cos ^{2} \phi\right] \boldsymbol{b} \otimes \boldsymbol{b}\right. \\
& +\left(a_{1}-a_{3}\right)\left(a_{2}-v^{2}\right) \sin \alpha \cos \alpha \boldsymbol{b} \otimes \boldsymbol{a}-v^{2}\left(a_{1}-a_{3}\right) \sin \alpha \cos \alpha \boldsymbol{a} \otimes \boldsymbol{b} \\
& +\left[\left(a_{1} a_{3}-\left(a_{2}-v^{2}\right)\left(d-v^{2}\right)-v^{2}\left(a_{1}+a_{3}-d\right)\right) \sin ^{2} \phi\right. \\
& \left.\left.+\left(a_{1} a_{3}-d\left(a_{2}-v^{2}\right)\right) \cos ^{2} \phi\right] \boldsymbol{a} \otimes \boldsymbol{a}\right\} .
\end{aligned}
$$

According to (6), tensors $Q$ and $S$ can be found by averaging over angle $\phi$ of expressions (35) and (36). Inasmuch as expression (36) is proportional to products of only odd powers of $\sin \phi$ and $\cos \phi$, then $S=0$. At the same time,

$$
\begin{aligned}
Q=-\frac{1}{v^{2}}\left\{\left(a_{2}-v^{2}\right)\left(d-v^{2}\right) J_{02}+\left[a_{1} a_{3}-v^{2}\left(a_{1}+a_{3}-d\right)\right] J_{20}\right\} \boldsymbol{b} \otimes \boldsymbol{b} \\
\quad-\left(a_{1}-a_{3}\right) \sin \alpha \cos \alpha J_{20}(\boldsymbol{b} \otimes \boldsymbol{a}+\boldsymbol{a} \otimes \boldsymbol{b})+\left[\left(d-v^{2}\right) J_{02}+d J_{20}\right] \boldsymbol{a} \otimes \boldsymbol{a},
\end{aligned}
$$


where

$\left(J_{02} ; J_{20}\right)=\frac{1}{\pi} \int_{0}^{\pi} \frac{\left(\sin ^{2} \phi ; \cos ^{2} \phi\right) \mathrm{d} \phi}{A \cos ^{4} \phi+B \sin ^{2} \phi \cos ^{2} \phi+C \sin ^{4} \phi}=\frac{1}{\pi} \int_{-\infty}^{+\infty} \frac{\left(1 ; x^{2}\right) \mathrm{d} x}{A x^{4}+B x^{2}+C}$.

Calculating the integrals, we obtain

$$
J_{02}=\frac{1}{\sqrt{C(B+2 \sqrt{A C})}}, \quad J_{20}=\frac{1}{\sqrt{A(B+2 \sqrt{A C})}} .
$$

Substituting values $A, B, C$ (34) into (38) and then (38) into (37), we find the final expression for the tensor $Q$

$$
\begin{aligned}
Q= & \frac{1}{\sqrt{\Delta(v)}}\left\{-\frac{1}{v^{2}}\left[\sqrt{\left(a_{2}-v^{2}\right)\left(d-v^{2}\right)}+\frac{1}{\sqrt{a_{1} a_{3}}}\left[a_{1} a_{3}-v^{2}\left(a_{1}+a_{3}-d\right)\right]\right] \boldsymbol{b} \otimes \boldsymbol{b}\right. \\
& \left.-\frac{1}{\sqrt{a_{1} a_{3}}}\left(a_{1}-a_{3}\right) \sin \alpha \cos \alpha(\boldsymbol{b} \otimes \boldsymbol{a}+\boldsymbol{a} \otimes \boldsymbol{b})+\left(\sqrt{\frac{d-v^{2}}{a_{2}-v^{2}}}+\frac{d}{\sqrt{a_{1} a_{3}}}\right) \boldsymbol{a} \otimes \boldsymbol{a}\right\},
\end{aligned}
$$

where

$\Delta(v)=B+2 \sqrt{A C}=a_{1} a_{3}+a_{2} d-v^{2}\left(a_{1}+a_{3}\right)+2 \sqrt{a_{1} a_{3}\left(a_{2}-v^{2}\right)\left(d-v^{2}\right)}$.

Then, determinant of tensor $Q$ is

$$
\bar{Q}_{t}=-\frac{1}{v^{2} \sqrt{a_{1} a_{3}}} \sqrt{\frac{d-v^{2}}{a_{2}-v^{2}}},
$$

whereupon it is easy to obtain the pseudoinverse tensor $Q^{-}$and the surface impedance tensor $\gamma$ for the biaxial crystal with the cut plane coinciding with its symmetry plane

$$
\begin{aligned}
\gamma=-\frac{\mathrm{i}}{v} Q^{-}= & \frac{\mathrm{i}}{v \sqrt{\Delta(v)}}\left\{v^{2}\left(d \sqrt{\frac{a_{2}-v^{2}}{d-v^{2}}}+\sqrt{a_{1} a_{3}}\right) \boldsymbol{b} \otimes \boldsymbol{b}\right. \\
& +v^{2}\left(a_{1}-a_{3}\right) \sqrt{\frac{a_{2}-v^{2}}{d-v^{2}}} \sin \alpha \cos \alpha(\boldsymbol{b} \otimes \boldsymbol{a}+\boldsymbol{a} \otimes \boldsymbol{b}) \\
& \left.-\left[\sqrt{a_{1} a_{3}}\left(a_{2}-v^{2}\right)+\sqrt{\frac{a_{2}-v^{2}}{d-v^{2}}}\left[a_{1} a_{3}-v^{2}\left(a_{1}+a_{3}-d\right)\right]\right] \boldsymbol{a} \otimes \boldsymbol{a}\right\} .
\end{aligned}
$$

For the isotropic medium contacting with the biaxial crystal, tensor $S^{\prime}$ is zero and $Q^{\prime}$ is obtained from $Q$ by changing in (39) values $a_{1}, a_{2}, a_{3}, d$ to the inverse permittivity $a^{\prime}=\varepsilon^{\prime-1}$

$$
Q^{\prime}=-\frac{1}{v^{2}} \sqrt{\frac{a^{\prime}-v^{2}}{a^{\prime}}} \boldsymbol{b} \otimes \boldsymbol{b}+\frac{1}{\sqrt{a^{\prime}\left(a^{\prime}-v^{2}\right)}} \boldsymbol{a} \otimes \boldsymbol{a} .
$$

Then, according to the second formula of (5), the surface impedance tensor of the isotropic medium equals

$$
\gamma^{\prime}=\frac{\mathrm{i}}{v} Q^{\prime-}=-\mathrm{i} v \sqrt{\frac{a^{\prime}}{a^{\prime}-v^{2}}} \boldsymbol{b} \otimes \boldsymbol{b}+\frac{\mathrm{i}}{v} \sqrt{a^{\prime}\left(a^{\prime}-v^{2}\right)} \boldsymbol{a} \otimes \boldsymbol{a} .
$$

In the next section, we examine the tensor $\gamma$ at $v \rightarrow v_{\mathrm{L}}$ in order to establish the necessary existence conditions for surface polaritons at the interface of biaxial crystals and isotopic media according to (9). 


\section{Surface impedance tensor $\gamma$ at $\nu \rightarrow \nu_{\mathrm{L}}$}

Now we consider function $\Delta(v)$ (40) contained in expression (41) for the tensor $\gamma$. It is evident that it is a decreasing function on the interval $\left[0, v_{L}\right]$, and that $\Delta(0)>0$. As was established in section 4 , the value of the limiting frequency $v_{\mathrm{L}}$ depends on the relations between material parameters $a_{1}, a_{2}, a_{3}$ (see table 1). If $v_{\mathrm{L}}=\sqrt{d}$ or $v_{\mathrm{L}}=\sqrt{a_{2}}$, then the value $\Delta\left(v_{\mathrm{L}}\right)$ is proportional with a coefficient $\left|a_{2}-d\right|^{-1}$ to the multiplier near $m_{q}^{2}$ in equation (21) or (22) of the part of the outer curve of the refraction surface section. But as is shown in section 3, this multiplier is positive in all cases listed in table 1, except (ii(b)). Therefore, $\Delta\left(v_{\mathrm{L}}\right)>0$ for the indicated values of $v_{\mathrm{L}}$.

If the relations $(\mathrm{ii}(\mathrm{b}))$ are satisfied, then $v_{\mathrm{L}}=\widetilde{\nu}_{\mathrm{L}}$ (see formula (27)). We prove by contradiction that $\Delta\left(\widetilde{v}_{\mathrm{L}}\right)=0$. Suppose $\Delta\left(\widetilde{v}_{\mathrm{L}}\right)=B+2 \sqrt{A C} \neq 0$, then $B^{2}-4 A C \neq 0$. But the quantity $B^{2}-4 A C$ at $v=\widetilde{v}_{\mathrm{L}}$ coincides with the left-hand side of (26) for $\widetilde{v}_{\mathrm{L}}$, so we come to a contradiction. Thus, in this case, tensor $\gamma$ diverges at $v=v_{\mathrm{L}}$.

From equation $\Delta\left(\widetilde{v}_{\mathrm{L}}\right)=0$, it follows that

$$
\sqrt{a_{1} a_{3}\left(a_{2}-\widetilde{v}_{\mathrm{L}}^{2}\right)\left(d-\widetilde{v}_{\mathrm{L}}^{2}\right)}=-\frac{1}{2}\left[a_{1} a_{3}+a_{2} d-\widetilde{v}_{\mathrm{L}}^{2}\left(a_{1}+a_{3}\right)\right]
$$

Now we apply necessary condition (9) of surface polariton existence at the interface of crystal and isotropic medium. The left-hand side of relation (9) is determined by the coefficient of dyad $\boldsymbol{a} \otimes \boldsymbol{a}$ in (41). So,

$$
\lim _{v \rightarrow v_{\mathrm{L}}} g(v) \sqrt{\frac{a_{2}-v^{2}}{d-v^{2}}}<0,
$$

where

$$
g(v)=a_{1} a_{3}-v^{2}\left(a_{1}+a_{3}-d\right)+\sqrt{a_{1} a_{3}\left(a_{2}-v^{2}\right)\left(d-v^{2}\right)}
$$

is a decreasing function on the interval $\left[0, v_{\mathrm{L}}\right]$. Next, we examine for which kind of relations listed in table 1 the inequality (44) is satisfied.

Relations (i) and (ii(a)), $v_{\mathrm{L}}=\sqrt{d}$. It is necessary to investigate the sign of the function $g(v)$ which is multiplied in (44) by the diverging coefficient $\left(d-v^{2}\right)^{-\frac{1}{2}}$. If $\alpha \neq 0$, we have

$$
g\left(v_{\mathrm{L}}\right)=g(\sqrt{d})=-\left(a_{1}-d\right)\left(d-a_{3}\right)<0 .
$$

Consequently, inequality (44) is satisfied.

Relation (ii(b)), $v_{\mathrm{L}}=\widetilde{v}_{\mathrm{L}}$. Supposing that $\alpha \neq \alpha_{2}$, we take into account inequality (28) and equation (43):

$$
\begin{aligned}
g\left(\widetilde{v}_{\mathrm{L}}\right)= & a_{1} a_{3}-\widetilde{v}_{\mathrm{L}}^{2}\left(a_{1}+a_{3}-d\right)-\frac{1}{2}\left(a_{1} a_{3}+a_{2} d\right)+\frac{1}{2} \widetilde{v}_{\mathrm{L}}^{2}\left(a_{1}+a_{3}\right) \\
= & a_{1} a_{3}+\widetilde{v}_{\mathrm{L}}^{2} d-\frac{1}{2}\left(a_{1} a_{3}+a_{2} d\right)-\frac{1}{2} \widetilde{v}_{\mathrm{L}}^{2}\left(a_{1}+a_{3}\right) \\
& <\frac{1}{2}\left(a_{1} a_{3}+a_{2} d\right)-\frac{1}{2} \widetilde{v}_{\mathrm{L}}^{2}\left(a_{1}+a_{3}\right)=-\sqrt{a_{1} a_{3}\left(a_{2}-\widetilde{v}_{\mathrm{L}}^{2}\right)\left(d-\widetilde{v}_{\mathrm{L}}^{2}\right)} \leqslant 0 .
\end{aligned}
$$

Thus, in this case, inequality (44) is satisfied for $\alpha \in\left[\alpha_{1}, \alpha_{2}\right)$.

Relations (ii(c)) and (iii), $v_{\mathrm{L}}=\sqrt{a_{2}}$. Inequality (44) is not satisfied because its left-hand side vanishes. This means that in case (ii(c)) or (iii) excitation of the surface polaritons is impossible. Specifically, a surface wave cannot propagate along a cut plane of the negative uniaxial crystal $\left(a_{1}>a_{3}=a_{2}\right)$ with optical axis situated in this plane. This fact was first established in [3, 4].

Thus, condition (9) is in essence a prohibitive condition and it allows one, without preliminary solving the dispersion equation, to establish relations of material parameters of an anisotropic medium, so as to forbid surface waves. For surface waves in symmetry planes of 
a biaxial crystal, inequality (9) leads to the following necessary (but not sufficient) condition of wave existence. The inverse permittivity $a_{2}$ referring to the crystallographic axis along the interface normal $\boldsymbol{q}$ should be greater than the other inverse permittivities $a_{1}$ and $a_{3}$ for axes in the symmetry plane.

\section{Analysis of the dispersion equation solutions}

The dispersion equation for the surface polaritons at the interface of a biaxial crystal and an isotropic medium is obtained by substituting expressions (41) and (42) for $\gamma$ and $\gamma^{\prime}$ in equation (4). It takes the form

$$
F(v)=0,
$$

where

$$
\begin{gathered}
F(v)=a^{\prime}+\sqrt{a_{1} a_{3}} \sqrt{\frac{a_{2}-v^{2}}{d-v^{2}}}+\frac{1}{\sqrt{\Delta(v)}}\left[g(v) \sqrt{\frac{a_{2}-v^{2}}{d-v^{2}}} \sqrt{\frac{a^{\prime}}{a^{\prime}-v^{2}}}\right. \\
\left.+\left(d \sqrt{\frac{a_{2}-v^{2}}{d-v^{2}}}+\sqrt{a_{1} a_{3}}\right) \sqrt{a^{\prime}\left(a^{\prime}-v^{2}\right)}\right]
\end{gathered}
$$

and functions $\Delta(v)$ and $g(v)$ are defined by (40) and (45), respectively. The dispersion equation describes surface polaritons for any cut planes of the biaxial crystal parallel to the symmetry planes provided that $a_{1}, a_{2}, a_{3}, a^{\prime}$ are positive.

It is obvious that $F(0)>0$. Equation (46) has a unique solution $v=v_{\mathrm{S}}$ only if the function $F(v)$ changes its sign on the interval $\left(0, \hat{v}_{\mathrm{L}}\right)$, i.e. if

$$
\lim _{\nu \rightarrow \hat{v}_{\mathrm{L}}} F(v)<0,
$$

where $\hat{v}_{\mathrm{L}}=\min \left(v_{\mathrm{L}}, v_{\mathrm{L}}^{\prime}\right), v_{\mathrm{L}}^{\prime}=\sqrt{a^{\prime}}$ is a limiting frequency of surface waves in the isotropic medium. Thus, condition (48) is a necessary and sufficient existence condition for surface electromagnetic waves. If it is satisfied, then at a given frequency $\omega$, the wave vector projection $k_{\mathrm{S}}=\omega /\left(c v_{\mathrm{S}}\right)$ to the $\boldsymbol{b}$-direction can be found with the use of solution $v=v_{\mathrm{S}}$ of (46) and (47).

According to the relations between material parameters $a_{1}, a_{2}, a_{3}, a^{\prime}$, the limiting frequency $\hat{v}_{\mathrm{L}}$ can take one of the following values: $\sqrt{a^{\prime}}, \sqrt{d}$ or $\widetilde{v}_{\mathrm{L}}$ (the case $\hat{v}_{\mathrm{L}}=\sqrt{a_{2}}$ is eliminated from consideration in view of previous section's results). Now we establish further restrictions of material parameters arising from condition (48).

Let $\hat{v}_{\mathrm{L}}=\sqrt{a^{\prime}}$. Then, at the limit $v \rightarrow \hat{v}_{\mathrm{L}}$, function $F(v)$ diverges owing to coefficient $\sqrt{a^{\prime} /\left(a^{\prime}-v^{2}\right)}$ of $g(v)($ see $(47))$, so inequality (48) is identical to $g\left(\sqrt{a^{\prime}}\right)<0$ or

$$
a_{1} a_{3}-a^{\prime}\left(a_{1}+a_{3}-d\right)+\sqrt{a_{1} a_{3}\left(a_{2}-a^{\prime}\right)\left(d-a^{\prime}\right)}<0 .
$$

If $a^{\prime} \leqslant a_{3}$, then inequality (49) is not satisfied:

$$
g\left(\sqrt{a^{\prime}}\right) \geqslant g\left(\sqrt{a_{3}}\right)=a_{3}\left(d-a_{3}\right)+\sqrt{a_{1} a_{3}\left(a_{2}-a_{3}\right)\left(d-a_{3}\right)} \geqslant 0 .
$$

Let $\hat{v}_{\mathrm{L}}=\sqrt{d}$. The function $F(v)$ at $v \rightarrow \hat{v}_{\mathrm{L}}$ diverges owing to the coefficients $\sqrt{\left(a_{2}-v^{2}\right) /\left(d-v^{2}\right)}$. Then, condition (48) is equivalent to $\sqrt{a_{1} a_{3}}+$ $[\Delta(\sqrt{d})]^{-\frac{1}{2}}\left[g(\sqrt{d}) \sqrt{a^{\prime} /\left(a^{\prime}-d\right)}+d \sqrt{a^{\prime}\left(a^{\prime}-d\right)}\right]<0$ or

$-\sqrt{a^{\prime}}\left[a_{1} a_{3}-d\left(a_{1}+a_{3}-a^{\prime}\right)\right]>\sqrt{a_{1} a_{3}\left[a_{1} a_{3}-d\left(a_{1}+a_{3}-a_{2}\right)\right]\left(a^{\prime}-d\right)}$. 
Table 2. Relations between material parameters of the contacting media for which the dispersion equation does not have any solution.

\begin{tabular}{|c|c|c|c|c|}
\hline Case & $\begin{array}{l}\text { Relations } \\
\text { between } a_{1}, a_{2}, a_{3}\end{array}$ & Angle $\alpha$ & Parameter $a^{\prime}$ & $\begin{array}{l}\text { Reference to condition } \\
\text { not satisfied }\end{array}$ \\
\hline (i) & $a_{2} \geqslant a_{1}>a_{3}$ & & $\begin{array}{l}a^{\prime} \leqslant a_{3} \\
a^{\prime} \geqslant a_{1}\end{array}$ & $\begin{array}{l}(49) \\
(50)\end{array}$ \\
\hline (ii(a)) & $a_{1}>a_{2}>a_{3}$ & $0 \leqslant \alpha<\alpha_{1}$ & $\begin{array}{l}a^{\prime} \leqslant a_{3} \\
a^{\prime} \geqslant a_{2}\end{array}$ & $\begin{array}{l}(49) \\
(50)\end{array}$ \\
\hline (ii(b)) & $a_{1}>a_{2}>a_{3}$ & $\alpha_{1} \leqslant \alpha \leqslant \alpha_{2}$ & $\begin{array}{l}a^{\prime} \leqslant a_{3} \\
a^{\prime} \geqslant a_{2}\end{array}$ & $\begin{array}{l}(49) \\
(51)\end{array}$ \\
\hline $\begin{array}{l}\text { (ii(c)) } \\
\text { (iii) }\end{array}$ & $\begin{array}{l}a_{1}>a_{2}>a_{3} \\
a_{1}>a_{3} \geqslant a_{2}\end{array}$ & $\alpha_{2}<\alpha \leqslant \frac{\pi}{2}$ & $\begin{array}{l}\text { Any } \\
\text { Any }\end{array}$ & $\begin{array}{l}(44) \\
(44)\end{array}$ \\
\hline
\end{tabular}

In the case (i) (see table 1) and, in addition, $a^{\prime} \geqslant a_{1}$, the relation (50) is not satisfied. Indeed, in this case, the left-hand side of (50) is non-positive:

$-\sqrt{a^{\prime}}\left[a_{1} a_{3}-d\left(a_{1}+a_{3}-a^{\prime}\right)\right] \leqslant-\sqrt{a^{\prime}}\left[a_{1} a_{3}-d\left(a_{1}+a_{3}-a_{1}\right)\right]=-\sqrt{a^{\prime}}\left(a_{1}-d\right) a_{3} \leqslant 0$.

Inequality (50) is also not satisfied if the material parameters yield to relations (ii(a)) and $a^{\prime} \geqslant a_{2}$ :

$$
-\sqrt{a^{\prime}}\left[a_{1} a_{3}-d\left(a_{1}+a_{3}-a^{\prime}\right)\right] \leqslant-\sqrt{a^{\prime}}\left[a_{1} a_{3}-d\left(a_{1}+a_{3}-a_{2}\right)\right]<0 .
$$

Here, it is taken into account that $a_{1} a_{3}-d\left(a_{1}+a_{3}-a_{2}\right)>0$ at $0 \leqslant \alpha<\alpha_{1}$ (section 3 ).

Finally, we examine the case $\hat{v}_{\mathrm{L}}=\widetilde{v}_{\mathrm{L}}$. Function $F(v)$ diverges at $v \rightarrow \hat{v}_{\mathrm{L}}$ owing to vanishing of the value $\Delta\left(\widetilde{v}_{\mathrm{L}}\right)$. Thus, a necessary and sufficient existence condition (48) is fulfilled only if expression in square brackets in (47) is negative for $v=\hat{v}_{\mathrm{L}}$ :

$$
g\left(\widetilde{v}_{\mathrm{L}}\right) \sqrt{\frac{a_{2}-\widetilde{v}_{\mathrm{L}}^{2}}{d-\widetilde{v}_{\mathrm{L}}^{2}}} \sqrt{\frac{a^{\prime}}{a^{\prime}-\widetilde{v}_{\mathrm{L}}^{2}}}+\left(d \sqrt{\frac{a_{2}-\widetilde{v}_{\mathrm{L}}^{2}}{d-\widetilde{v}_{\mathrm{L}}^{2}}}+\sqrt{a_{1} a_{3}}\right) \sqrt{a^{\prime}\left(a^{\prime}-\widetilde{v}_{\mathrm{L}}^{2}\right)}<0 .
$$

Inequality (51) is greatly reduced owing to equations (26) and (43). Multiplying both parts of (51) by $\left(d-\widetilde{v}_{\mathrm{L}}^{2}\right) \sqrt{a_{1} a_{3}\left(a^{\prime}-\widetilde{v}_{\mathrm{L}}^{2}\right) / a^{\prime}}$, we obtain

$a_{1} a_{3}\left(d-\widetilde{v}_{\mathrm{L}}^{2}\right)\left(a_{2}+a^{\prime}-2 \widetilde{v}_{\mathrm{L}}^{2}\right)+\left[a_{1} a_{3}+a^{\prime} d-\widetilde{v}_{\mathrm{L}}^{2}\left(a_{1}+a_{3}\right)\right] \sqrt{a_{1} a_{3}\left(a_{2}-\widetilde{v}_{\mathrm{L}}^{2}\right)\left(d-\widetilde{v}_{\mathrm{L}}^{2}\right)}<0$.

After equation (43) is substituted, we have

$$
\begin{gathered}
\left(a_{1}-a_{3}\right)^{2} \widetilde{v}_{\mathrm{L}}^{4}- \\
{\left[\left(a_{1}+a_{3}\right)\left(2 a_{1} a_{3}+a_{2} d+a^{\prime} d\right)-2 a_{1} a_{3}\left(a_{2}+a^{\prime}\right)-4 a_{1} a_{3} d\right] \widetilde{v}_{\mathrm{L}}^{2}} \\
+\left(a_{1} a_{3}+a_{2} d\right)\left(a_{1} a_{3}+a^{\prime} d\right)-2 a_{1} a_{3} d\left(a_{2}+a^{\prime}\right)>0 .
\end{gathered}
$$

In the end, using (26) and eliminating $\widetilde{v}_{\mathrm{L}}^{4}$ from (52), we obtain

$$
\left(a_{2}-a^{\prime}\right)\left\{\left(a_{1} a_{3}-a_{2} d\right) d-\widetilde{v}_{\mathrm{L}}^{2}\left[2 a_{1} a_{3}-d\left(a_{1}+a_{3}\right)\right]\right\}>0 .
$$

The multiplier in curly braces is positive owing to inequalities (28) and $a_{1} a_{3}-d\left(a_{1}+a_{3}-a_{2}\right)<$ 0 for angles $\alpha$ in the range $\left(\alpha_{1}, \alpha_{2}\right)$ (case (ii(b)))

$\left(a_{1} a_{3}-a_{2} d\right) d-\widetilde{v}_{\mathrm{L}}^{2}\left[2 a_{1} a_{3}-d\left(a_{1}+a_{3}\right)\right]>\left(a_{1} a_{3}-a_{2} d\right) d-d\left[2 a_{1} a_{3}-d\left(a_{1}+a_{3}\right)\right]>0$.

Thus, condition (51) comes to a simple inequality $a^{\prime}<a_{2}$. One can show that we also obtain the same result from (48) if $\alpha=\alpha_{1}$. 


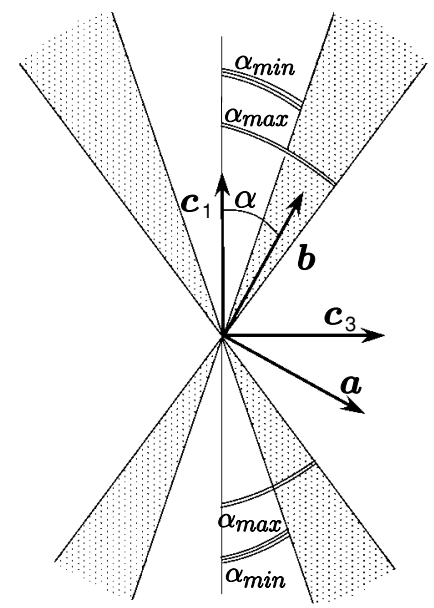

Figure 2. Sectors of allowed propagation directions $\boldsymbol{b}$ of surface polaritons.

To summarize our analysis, we present table 2. We indicate relations involving $a_{1}, a_{2}$, $a_{3}, a^{\prime}$, when the dispersion equation (46) does not have any solution and hence propagation of the surface wave is impossible. Evidently, the table does not include the relations

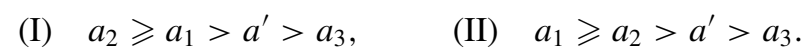

Only these relations for material parameters can satisfy the necessary and sufficient existence condition (48). However, it is possible not for all angles $\alpha$ (i.e., not for arbitrary propagation directions of surface polaritons) but only for

$$
\alpha_{\min }<\alpha<\alpha_{\max }
$$

where

$$
\begin{aligned}
\sin ^{2} \alpha_{\min }= & \frac{a_{3}\left(a^{\prime}-a_{3}\right)\left[a_{1}\left(a_{2}-a_{3}\right)-a^{\prime}\left(a^{\prime}-a_{3}\right)\right]}{\left(a_{1}-a_{3}\right)\left[a^{\prime 3}-2\left(a_{1}+a_{3}\right) a^{\prime 2}+\left(a_{1}+a_{3}\right)^{2} a^{\prime}-a_{1} a_{3}\left(a_{1}-a_{2}+a_{3}\right)\right]}, \\
\sin ^{2} \alpha_{\max }= & \frac{1}{2\left(a_{1}-a_{3}\right) a^{\prime 2}}\left\{a_{1}\left[a_{2} a_{3}-a^{\prime}\left(3 a_{3}-2 a^{\prime}\right)\right]\right. \\
& \left.\quad-\sqrt{a_{1} a_{3}\left(a_{2}-a^{\prime}\right)\left[a_{1} a_{2} a_{3}-5 a_{1} a_{3} a^{\prime}+4\left(a_{1}+a_{3}\right) a^{\prime 2}-4 a^{\prime 3}\right]}\right\} .
\end{aligned}
$$

Expressions (55) and (56) for limiting angles $\alpha_{\min }$ and $\alpha_{\max }$ are obtained by changing inequalities (50) and (49) to equalities and taking into account (16). As for condition (51), as stated above, it does not lead to any restriction of $\alpha$. It is reduced to inequality $a^{\prime}<a_{2}$ which is satisfied when (54) takes place.

Dispersion equation (46) does not change its form when $\alpha$ is replaced by $-\alpha$ or $\pi-\alpha$. Thus, the boundary plane of a biaxial crystal and an isotropic medium contains four sectors of allowed propagation directions $\boldsymbol{b}$ of surface polaritons (figure 2). They are determined by angles $\alpha$ in intervals $\left(\alpha_{\min }, \alpha_{\max }\right),\left(\pi-\alpha_{\max }, \pi-\alpha_{\min }\right),\left(\alpha_{\min }-\pi, \alpha_{\max }-\pi\right),\left(-\alpha_{\max },-\alpha_{\min }\right)$.

Now we investigate dependence of limiting angles $\alpha_{\min }$ and $\alpha_{\max }$ on the inverse permittivity $a^{\prime}$ of isotropic medium when parameters $a_{1}, a_{2}, a_{3}$ of biaxial crystal are fixed. It is easy to see that

$$
\lim _{a^{\prime} \rightarrow a_{3}} \alpha_{\min }=\lim _{a^{\prime} \rightarrow a_{3}} \alpha_{\max }=0,
$$




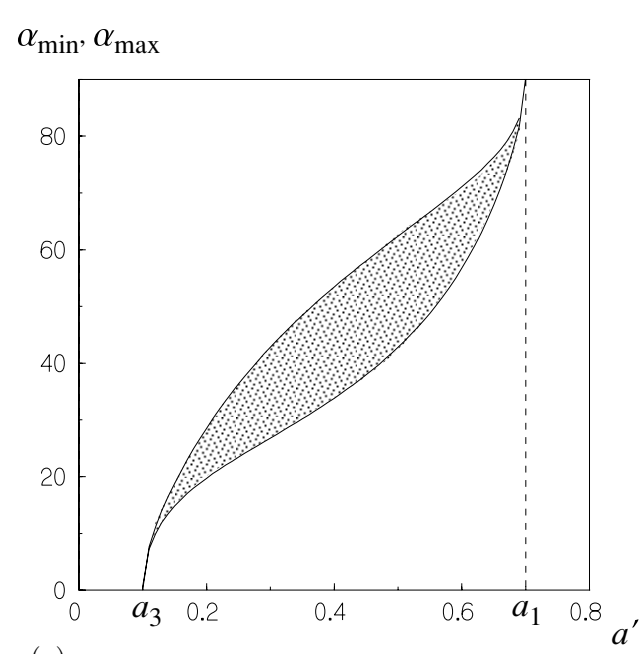

(a)

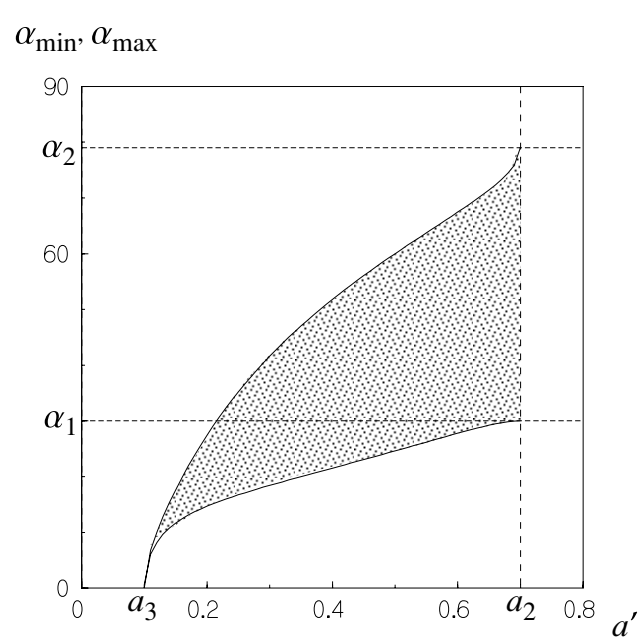

(b)

Figure 3. Dependence of limiting angles $\alpha_{\min }$ and $\alpha_{\max }$ on the inverse permittivity $a^{\prime}$ of isotropic medium: (a) $a_{1}=0.7, a_{2}=0.9, a_{3}=0.1 ;(b) a_{1}=0.9, a_{2}=0.7, a_{3}=0.1$.

i.e., when $a^{\prime} \rightarrow a_{3}$, existence sectors are situated close to the crystal axis given by the unit vector $c_{1}$ and their angular width is vanishingly small.

Assume that relations (I) for the parameters (see (54)) occur. Then, parameter $a^{\prime}$ cannot exceed $a_{1}$ and

$$
\lim _{a^{\prime} \rightarrow a_{1}} \alpha_{\min }=\lim _{a^{\prime} \rightarrow a_{1}} \alpha_{\max }=\frac{\pi}{2} .
$$

Here, at $a^{\prime} \rightarrow a_{1}$, angular width of the sectors also becomes small, and they are situated close to the crystal axis given by the unit vector $c_{3}$. Typical plots of dependence of angles $\alpha_{\min }$ and $\alpha_{\max }$ versus $a^{\prime}$ for that case are shown in figure $3(a)$.

The case of material parameter relations (II) when optical axes of the biaxial crystal are situated in the interface plane is of more interest. Here, $a^{\prime}$ is arranged between $a_{3}$ and $a_{2}$, and when $a^{\prime} \rightarrow a_{3}$ equations (57) are satisfied as before. However, it is easy to establish that

$$
\lim _{a^{\prime} \rightarrow a_{2}} \alpha_{\min }=\alpha_{1}, \quad \lim _{a^{\prime} \rightarrow a_{2}} \alpha_{\max }=\alpha_{2}
$$

and in doing so $\alpha_{\min }<\alpha_{1}, \alpha_{\max }<\alpha_{2}$, where the angles $\alpha_{1}$ and $\alpha_{2}$ are determined by formulae (24). If value $a^{\prime}$ is just a little smaller than $a_{2}$, then the existence interval practically coincides with $\left(\alpha_{1}, \alpha_{2}\right)$. Note that the optical axis of the crystal with position characterized by angle $\alpha_{0}$ (23) lies in the corresponding sector of the allowed propagation directions since $\alpha_{1}<\alpha_{0}<\alpha_{2}$. As soon as $a^{\prime}$ becomes equal to or exceeds parameter $a_{2}$, existence intervals having finite angular widths at $a^{\prime} \lesssim a_{2}$ disappear totally and surface wave cannot propagate in any direction in the interface plane. The reason is that for all $\alpha \in\left(\alpha_{1}, \alpha_{2}\right)$, condition (53) now fails to operate. This case is illustrated by figure 3(b). When $a^{\prime}=a_{2}$, a wave in biaxial crystal fails to be localized close to the interface for entire range of $\alpha \in\left(\alpha_{1}, \alpha_{2}\right)$ (coefficients $\eta_{1}$ and $\eta_{2}$ in (1) become real and $\eta_{1}=-\eta_{2}$; that corresponds to the total internal reflection of the body wave in the biaxial crystal from the boundary of the isotropic medium).

Let us recall that relations (54) and expressions (55) and (56) are obtained with the assumption that the normal $\boldsymbol{q}$ to the interface plane coincides with $c_{2}$, and $a_{1}>a_{3}$ (see section 3). Thus, as follows from (54), it is necessary and sufficient for existence of the 


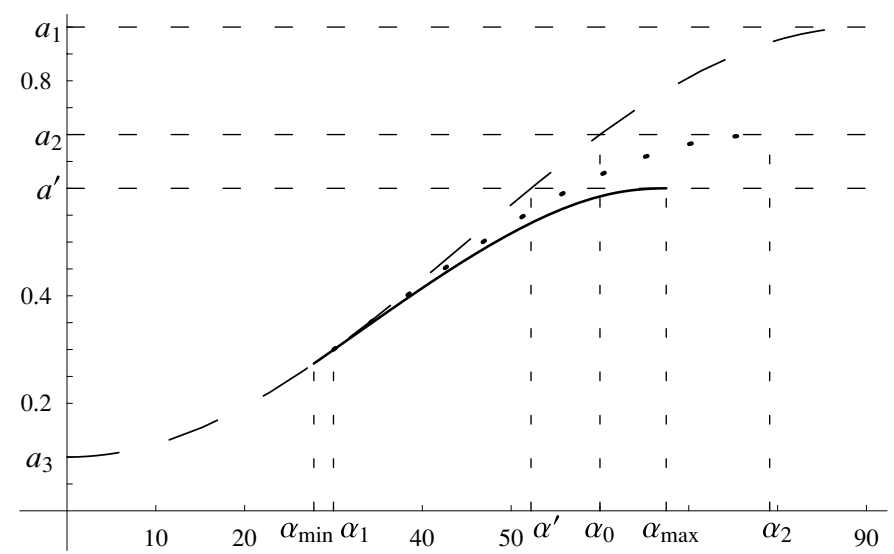

Figure 4. Solid line shows $v_{\mathrm{S}}^{2}=v_{\mathrm{S}}^{2}(\alpha)$ when $a_{1}=0.9, a_{2}=0.7, a_{3}=0.1, a^{\prime}=0.6$; dashed and doted lines show $d=d(\alpha)=a_{1} \sin ^{2} \alpha+a_{3} \cos ^{2} \alpha$ and $\widetilde{v}_{\mathrm{L}}^{2}=\widetilde{v}_{\mathrm{L}}^{2}(\alpha)$, respectively. Angles $\alpha_{0}, \alpha_{1}, \alpha_{2}, \alpha^{\prime}$ are calculated by formulae (23), (24) and (62), and $\alpha_{\min }, \alpha_{\max }$ by formulae (55) and (56).

surface electromagnetic waves that parameter $a_{3}$ be the smallest among $a_{1}, a_{2}, a_{3}, a^{\prime}$, and next ascending parameter be $a^{\prime}$.

It is clear that surface polaritons can be excited only in two symmetry planes of the same biaxial crystal. One of these planes includes optical axes of the crystal, and relations (54(II)) are satisfied. The other plane is perpendicular to the tensor $\varepsilon^{-1}$ principal axis which corresponds to the greatest among the inverse permittivities $a_{1}, a_{2}, a_{3}$. When coming from the first plane to the second plane, one needs to change $a_{1} \leftrightarrow a_{2}$ in all equations. Inequalities (II) in doing so are changed by (I).

Thus, conditions (54) establish existence of solutions of dispersion equation (46) and (47). These solutions can be found numerically. In figure 4, we show the plot of $v_{\mathrm{S}}^{2}$ as a function of the propagation direction angle $\alpha$ at fixed values of inverse permittivities in case of relations (II). Plots $d=d(\alpha)$ and $\widetilde{v}_{\mathrm{L}}^{2}=\widetilde{v}_{\mathrm{L}}^{2}(\alpha)$ (27) are also given which are used for finding the square of the limiting frequency of the surface waves.

Now we define the anisotropy parameters of the crystal $\chi_{1}=\left(a_{1}-a_{3}\right) / a_{3}, \chi_{2}=$ $\left(a_{2}-a_{3}\right) / a_{3}$ and the parameter for the isotropic medium $\chi^{\prime}=\left(a^{\prime}-a_{3}\right) / a_{3}$. Then, conditions (54) take the form

$$
\text { (I) } \chi_{2} \geqslant \chi_{1}>\chi^{\prime}>0, \quad \text { (II) } \chi_{1} \geqslant \chi_{2}>\chi^{\prime}>0
$$

and formulae (55) and (56) are written as

$$
\begin{aligned}
\sin ^{2} \alpha_{\min }= & \frac{\chi^{\prime}}{\chi_{1}}\left[1+\frac{\chi^{\prime}\left(\chi_{1}-\chi^{\prime}\right)^{2}}{\left(1+\chi_{1}\right)\left(\chi_{2}-\chi^{\prime}\right)+\chi^{\prime}\left(\chi_{1}-\chi^{\prime}\right)}\right]^{-1}, \\
\sin ^{2} \alpha_{\max }= & \frac{\chi^{\prime}}{\chi_{1}}+\frac{1}{2 \chi_{1}\left(1+\chi^{\prime}\right)^{2}}\left\{\left(1+\chi_{1}\right)\left(\chi_{2}-\chi^{\prime}\right)+2 \chi^{\prime}\left(1+\chi^{\prime}\right)\left(\chi_{1}-\chi^{\prime}\right)\right. \\
& \left.-\sqrt{\left(1+\chi_{1}\right)\left(\chi_{2}-\chi^{\prime}\right)\left[\left(1+\chi_{1}\right)\left(\chi_{2}-\chi^{\prime}\right)+4 \chi^{\prime}\left(1+\chi^{\prime}\right)\left(\chi_{1}-\chi^{\prime}\right)\right]}\right\} .
\end{aligned}
$$

We dwell on a case of small parameters $\chi_{1}$ and $\chi_{2}$ which is important for practical purposes when natural or artificial anisotropic materials (for example, photoelastic ones [20, 21]) are considered. Then, expressions (59) and (60) can be expanded to the first approximation by 
$\chi_{1}, \chi_{2}$ and $\chi^{\prime}$ to have the form

$$
\sin ^{2} \alpha_{\min , \max } \approx \frac{\chi^{\prime}}{\chi_{1}}\left[1 \mp \frac{\chi^{\prime}\left(\chi_{1}-\chi^{\prime}\right)^{2}}{\left(1+\chi_{1}\right)\left(\chi_{2}-\chi^{\prime}\right)+\chi^{\prime}\left(\chi_{1}-\chi^{\prime}\right)}\right] .
$$

The fraction denominator in square brackets in (61) can be presented as a sum of terms $\chi_{2}-\chi^{\prime}$ which is linear with respect to small anisotropy parameters and other quadratic terms $\chi_{1}\left(\chi_{2}-\chi^{\prime}\right)+\chi^{\prime}\left(\chi_{1}-\chi^{\prime}\right)$. These quadratic terms become essential when parameter $\chi^{\prime}$ is near to $\chi_{2}\left(\chi^{\prime} \lesssim \chi_{2}\right.$ or $\left.a^{\prime} \lesssim a_{2}\right)$. Note that in [11] when approximate formulae for angles $\alpha_{\min }$ and $\alpha_{\max }$ were derived, only the linear term $\chi_{2}-\chi^{\prime}$ was obtained.

It is clear from (61) that the greater the crystal anisotropy, the greater the angular width $\alpha_{\max }-\alpha_{\min }$ of existence sectors turns out to be (the situation is typical for singular surface polaritons in anisotropic media). The position of existence sectors in the interface plane is described by a central angle $\alpha^{\prime}$

$$
\sin \alpha^{\prime}=\sqrt{\frac{\chi^{\prime}}{\chi_{1}}}=\sqrt{\frac{a^{\prime}-a_{3}}{a_{1}-a_{3}}} .
$$

In figure 4 , this angle corresponds to the point of crossing the plot $d=d(\alpha)$ and horizontal line with ordinate $a^{\prime}$.

\section{Interface of uniaxial crystal and isotropic medium}

If one of the inverse permittivities $a_{1}$ or $a_{3}$ coincides with $a_{2}$, then we come to study the surface electromagnetic waves at the interface of the isotropic medium and the uniaxial crystal with the optical axis in the interface plane. This event was first analysed in [3, 4]. As was noted above, surface waves are impossible at the cuts of negative crystals when $a_{1}>a_{3}=a_{2}$. At the same time, for positive crystals, relations (54) take the form $a_{2}=a_{1}>a^{\prime}>a_{3}$. From formula (23), it follows that for such crystals $\alpha_{0}=\frac{\pi}{2}$, i.e. the optical axis is directed along vector $c_{3}$ (see figure 2). So, for describing propagation of surface waves, it is convenient to use angle $\varphi=\frac{\pi}{2}-\alpha$ formed by vectors $\boldsymbol{b}$ and $\boldsymbol{c}_{3}$. As a result, from (55) and (56), the following expressions for limiting angles are obtained:

$$
\begin{aligned}
& \sin ^{2} \varphi_{\min }=1-\sin ^{2} \alpha_{\max }=\frac{\xi}{2}\left[1-\chi \xi+\sqrt{(1-\chi \xi)^{2}+4 \chi}\right] \\
& \sin ^{2} \varphi_{\max }=1-\sin ^{2} \alpha_{\min }=\frac{(1+\chi)^{3} \xi}{(1+\chi)^{2}(1+\chi \xi)-\chi^{2}(1-\xi)^{2}},
\end{aligned}
$$

where $\chi=\left(\varepsilon_{\mathrm{e}}-\varepsilon_{\mathrm{o}}\right) / \varepsilon_{\mathrm{o}}$ is the anisotropy parameter of the uniaxial crystal and $\xi=$ $\left(\varepsilon^{\prime}-\varepsilon_{\mathrm{o}}\right) /\left(\varepsilon_{\mathrm{e}}-\varepsilon_{\mathrm{o}}\right)$ is the parameter for the isotropic medium; $\varepsilon_{\mathrm{o}}$ and $\varepsilon_{\mathrm{e}}$ are the permittivities referring to ordinary and extraordinary waves, respectively. Expressions (63) and (64) were first obtained in [4].

\section{Conclusion}

The main result obtained above is in establishing the existence conditions (54) for singular surface polaritons, which propagate along the symmetry plane of the biaxial crystal contacting with the isotropic medium. The exact expressions (55) and (56) are obtained for limiting angles of the sectors of the allowed propagation directions. Surface polaritons can propagate only if relations (54(I)) or (54(II)) of 12 possible relations involving material parameters $a_{1}, a_{2}, a_{3}, a^{\prime}$ $\left(a_{1}>a_{3}\right)$ take place. When inequalities (II) are satisfied and the inverse permittivity of 
the isotropic medium $a^{\prime}$ continuously changes from $a_{3}$ to $a_{2}$, then the angular width of the existence sectors increases. As soon as $a^{\prime}$ exceeds $a_{2}$, these sectors vanish abruptly and entirely (figure $3(b)$ ). This fact may be used for constructing optical shutters based on surface electromagnetic waves.

With use of the methods developed in [14, 15], surface polaritons can analogously be studied for other plane cuts of biaxial crystal, for example, for those that contain only one of the symmetry axes. To derive the polariton dispersion equation for cut planes without any symmetry elements of the crystal, it is advisable to use systems of computer algebra.

The results obtained are also applicable when anisotropy is induced externally in one of the contacting media. For example, electro-optical cubic crystals of 23 and $\overline{4} 3 m$ symmetry classes are optically isotropic when an external electric field is absent. If the external field is applied, they become biaxial (Pockel's effect). Changing the electric intensity, one can dynamically vary the position of the surface polariton existence sectors at the interface of such a crystal and non-electro-optical isotropic medium.

\section{Acknowledgment}

The work has been supported by the Basic Research Foundation of Belarus, grant F04M-140.

\section{References}

[1] Baumberg J J 2002 Phys. World 15 37-41

[2] Agranovich V M 1982 Surface Polaritons ed V M Agranovich and D C Mills (Amsterdam: North-Holland)

[3] Marchevsky F N, Strizhevsky V L and Strizhevsky S V 1984 Fiz. Tverd. Tela 26 1501-3

[4] Dyakonov M I 1988 Zh. Eksp. Theor. Fiz. 94 119-23 Dyakonov M I 1988 Sov. Phys.-JETP 67 714-6 (Engl. Transl.)

[5] Furs A N and Barkovsky L M 2003 Zh. Tekh. Fiz. 73 9-16 Furs A N and Barkovsky L M 2003 Tech. Phys. 48 385-92 (Engl. Transl.)

[6] Barkovsky L M et al 2004 Études on Theoretical Physics ed L M Barkovsky, I D Feranchuk and Ya M Shnir (Singapore: World Scientific) p 283

[7] Averkiev N S and D'yakonov M I 1990 Opt. Spektrosk. 68 1118-21 Averkiev N S and D'yakonov M I 1990 Opt. Spectrosc. 68 653-6 (Engl. Transl.)

[8] Darinskii A N 2001 Kristallografiya 46 916-8 Darinskii A N 2001 Crystallogr. Rep. 46 842-4 (Engl. Transl.)

[9] Furs A N, Galynsky V M and Barkovsky L M 2005 Opt. Spektrosk. 98 497-503

[10] Alshits V I and Lyubimov V N 2002 Fiz. Tverd. Tela 44 371-4 Alshits V I and Lyubimov V N 2002 Phys. Solid State 44 386-90 (Engl. Transl.)

[11] Alshits V I and Lyubimov V N 2002 Fiz. Tverd. Tela 44 1895-9 Alshits V I and Lyubimov V N 2002 Phys. Solid State 44 1988-92 (Engl. Transl.)

[12] Chadwick P and Smith G D 1977 Foundations of the Theory of Surface Waves in Anisotropic Elastic Media (Adv. Appl. Mech. vol 17) ed C-S Yih (New York: Academic) p 303

[13] Furs A N and Barkovsky L M 1997 Microw. Opt. Technol. Lett. 14 301-5

[14] Furs A N and Barkovsky L M 1999 J. Opt. A: Pure Appl. Opt. 1 109-15

[15] Galynsky V M, Furs A N and Barkovsky L M 2004 J. Phys. A: Math. Gen. 37 5083-96

[16] Barkovsky L M, Borzdov G N and Lavrinenko A V 1987 J. Phys. A: Math. Gen. 20 1095-106

[17] Fedorov F I 1976 Theory of Gyrotropy (Minsk: Nauka i Teknika)

[18] Penrose R A 1955 Proc. Camb. Phil. Soc. 51 406-13

[19] Barkovsky L M and Furs A N 2003 Operator Methods of the Description of Optical Fields in Complex Media (Minsk: Belaruskaya Nauka)

[20] Coker E G and Filon L N G 1957 A Treatise on Photo-Elasticity (Cambridge: Cambridge University Press)

[21] Theocaris P S and Gdoutos E E 1979 Matrix Theory of Photoelasticity (Berlin: Springer) 